\title{
Antimicrobial Resistance Profile and Genotypic Characteristics of Streptococcus suis Capsular Type 2 Isolated from Clinical Carrier Sows and Diseased Pigs in China
}

\author{
Chunping Zhang, ${ }^{1}$ Zhongqiu Zhang, ${ }^{2}$ Li Song, ${ }^{1}$ Xuezheng Fan, \\ Fang Wen, ${ }^{1}$ Shixin $\mathrm{Xu},{ }^{1}$ and Yibao Ning $^{1}$ \\ ${ }^{1}$ National Antimicrobial Resistance Monitoring Laboratory in Bacteria of Animal Origin, \\ Department of Inspection Technology Research, China Institute of Veterinary Drug Control, Beijing 100081, China \\ ${ }^{2}$ China Animal Disease Control Center, Beijing 100125, China \\ Correspondence should be addressed to Yibao Ning; ningyibao@sohu.com
}

Received 11 June 2014; Revised 20 October 2014; Accepted 28 October 2014

Academic Editor: Madhab K. Chattopadhyay

Copyright ( 2015 Chunping Zhang et al. This is an open access article distributed under the Creative Commons Attribution License, which permits unrestricted use, distribution, and reproduction in any medium, provided the original work is properly cited.

\begin{abstract}
Streptococcus suis serotype 2 is an important zoonotic pathogen. Antimicrobial resistance phenotypes and genotypic characterizations of S. suis 2 from carrier sows and diseased pigs remain largely unknown. In this study, 96 swine S. suis type 2, 62 from healthy sows and 34 from diseased pigs, were analyzed. High frequency of tetracycline resistance was observed, followed by sulfonamides. The lowest resistance of $S$. suis 2 for $\beta$-lactams supports their use as the primary antibiotics to treat the infection of serotype 2 . In contrast, 35 of 37 S. suis 2 with $M_{L} S_{B}$ phenotypes were isolated from healthy sows, mostly encoded by the ermB and/or the $m e f A$ genes. Significantly lower frequency of $m r p+l e p f+/ s l y+$ was observed among serotype 2 from healthy sows compared to those from diseased pigs. Furthermore, isolates from diseased pigs showed more homogeneously genetic patterns, with most of them clustered in pulsotypes A and E. The data indicate the genetic complexity of S. suis 2 between herds and a close linkage among isolates from healthy sows and diseased pigs. Moreover, many factors, such as extensive use of tetracycline or diffusion of Tn916 with tetM, might have favored for the pathogenicity and widespread dissemination of S. suis serotype 2.
\end{abstract}

\section{Introduction}

Streptococcus suis is an important swine pathogen leading to big loss in pig production worldwide [1]. Among 35 serotypes currently identified, serotype 2 has gained more attention for the high prevalence and mortality rates in swine and human and is considered as an emerging zoonotic agent [2]. Furthermore, epidemiological surveillance has confirmed that $S$. suis type 2 can transmit from carrier pigs to humans $[3,4]$. Clinically healthy carrier sows, harboring S. suis type 2 , are considered as the major source of infection for their offspring [5]. Since the infection of S. suis type 2 commonly occurred among suckling and weaned piglets, it is essential to investigate the association of antimicrobial resistance profile and genotypic characteristicsof isolates from carrier sows and diseased pigs.

In the absence of effective vaccines to fight against S. suis, antimicrobial agents have become increasingly important in treating and controlling the infection of $S$. suis type 2. Of these, $\beta$-lactams, tetracyclines, sulphonamides, and macrolides are the most common antimicrobials used for the prevention and treatment of streptococcal infection in pig production. But inappropriate use of antibiotic has led to the development of resistance of $S$. suis to these drugs worldwide [6-9]. Furthermore, coresistance to tetracyclines and macrolides/lincosamides in human S. suis isolates was observed, and the relevance of Tn916-like conjugative transposon in coresistant mechanisms and clone diffusion have 
been studied $[9,10]$. However, to date, few reports about coresistance to these three classes of antibiotics and the Tn916 family were found in swine $S$. suis serotype 2 .

In recent years, research on $S$. suis type 2 has mainly concentrated on its potential virulence factors and pathogenic mechanisms. Many factors, including polysaccharide capsule (cps), muramidase-released protein (mrp), the extracellular protein factor $(e p f)$, the suilysin $(s l y)$, glyceraldehyde-3-phosphate dehydrogenase $(g d h)$, and a fibronectin/fibrinogen-binding protein $(f b p)$, were found to be associated with virulence of $S$. suis 2 [11-14]. Of these, mrp, epf, and sly were considered as the most relevant factors to the pathogenesis of S. suis by many researchers [15-17]. Recently, other new putative virulence factors, such as surface-associated subtilisin-like serine protease $(S s p A)$, factor H-binding protein $(f h b)$, have also been identified [18, 19]. However, our knowledge about pathogenesis of serotype 2 is still limited despite the increasing number of studies.

To deeply understand $S$. suis type 2 infection, molecular typing methods are usually applied to identify individual isolates and establish genotypic characterization. Currently, many typing methods, such as randomly amplified polymorphic DNA (RAPD) [20], ribotyping [21], multilocus sequences typing (MLST) $[22,23]$, and genome sequencing [24], have been used to define the diversity of S. suis and to distinguish virulent from nonvirulent isolates. Of these, pulsed-field gel electrophoresis (PFGE) is one of the most powerful molecular typing methods. Many researches had been done to compare differences between S. suis from different animals, and genetic diversity was found on the basis of isolates of the different pathogenic serotypes [25-27]. However, a thorough characterization of serotype 2 isolates from healthy sows and diseased pigs has not thus far been reported.

The aim of the study is to investigate antimicrobial resistance phenotypes, genotypes, and genetic patterns of S. suis type 2 from clinical carrier sows and diseased pigs. Furthermore, the phenotypic and genotypic characterizations of these isolates were compared. To the best of our knowledge, this is the first integrative report about resistance profile and genetic diversity of $S$. suis serotype 2 from clinically healthy sows and diseased pigs.

\section{Materials and Methods}

2.1. S. suis Type 2 Isolates. A total of 96 swine S. suis serotype 2 were included in this study. 62 isolates were recovered from tonsils of clinically healthy sows of 15 epidemiologically unrelated farms in 10 regions (Jiangsu, Sichuan, Guangdong, Guangxi, Anhui, Henan, Hebei, Jiangxi, Shandong, and Beijing) from March 2005 to November 2012. Of the 34 S. suis serotype 2 obtained from diseased pigs, 31 outbreak isolates were from 6 epidemiologically unrelated farms in Sichuan, Jiangsu, Anhui, Henan, and Beijing during 2005-2008, and three historical isolates (C55604, C55609, and C55612) were provided by China Veterinary Culture Collection Center (CVCC). All isolates were determined as S. suis types 2 by biochemical characteristics (API 20 strep, bioMérieux SA,
France) and sera agglutination reaction (special antisera provided by the Statens Serum Institut, Copenhagen, Denmark) and further confirmed as serotype 2 by positive PCR for the genes coding for the $16 \mathrm{~S}$ rRNA of $S$. suis and for the capsule of S. suis serotype 2 (cps2J) [23].

2.2. Antimicrobial Susceptibility. Antimicrobial susceptibility testing was performed using the standard broth microdilution method [28]. The following antimicrobial agents, the representatives of commonly used drug classes in China, were tested (with dilution ranges in parentheses): penicillin G $(0.06-8 \mu \mathrm{g} / \mathrm{mL})$, ampicillin $(0.12-16 \mu \mathrm{g} / \mathrm{mL})$, ceftiofur sodium $(0.12-16 \mu \mathrm{g} / \mathrm{mL})$, enrofloxacin $(0.015-4 \mu \mathrm{g} / \mathrm{mL})$, clindamycin $(0.03-4 \mu \mathrm{g} / \mathrm{mL})$, erythromycin $(0.06-8 \mu \mathrm{g} / \mathrm{mL})$, tilmicosin $(0.5-64 \mu \mathrm{g} / \mathrm{mL})$, chloramphenicol $(1-128 \mu \mathrm{g} / \mathrm{mL})$, tiamulin fumarate $(0.25-32 \mu \mathrm{g} / \mathrm{mL})$, tetracycline $(0.06-$ $8 \mu \mathrm{g} / \mathrm{mL})$, sulfisoxazole $(16-512 \mu \mathrm{g} / \mathrm{mL})$, and trimethoprim/ sulfamethoxazole $\quad(0.5 / 9.5-16 / 304 \mu \mathrm{g} / \mathrm{mL})$. Streptococcus pneumoniae ATCC 49619 was used as the quality control strain. The isolate was defined as an $\mathrm{MLS}_{\mathrm{B}}$-phenotype that was found to be resistant to erythromycin, lincomycin, and tilmicosin.

2.3. Genotyping. PCR virulence genotyping of all isolates was performed for the $m r p$, epf, sly, orf2, $f b p$, and $g d h$ genes. Moreover, 85 tetracycline-resistant $\left(\right.$ tet $\left.^{r}\right)$ isolates were also analyzed for the presence of tetracycline-resistant genes (tet $K$, tet $L$, tet $M$, and tet $O$ ), macrolide-resistant genes (ermB, erm $A$, and $m e f A)$, lincosamide-resistant gene $(\ln u B)$, and Tn916-like transposon family (intTn and xis), respectively. Positive and negative controls were included with each PCR assay. Target genes and the corresponding primer sequences were listed in Table 1. Confirmation of the amplicons was determined by DNA sequencing and the results were analyzed using BLAST software (http://www.ncbi.nlm.nih.gov).

2.4. PFGE Analysis. All S. suis serotype 2 isolates were typed using pulsed-field gel electrophoresis (PFGE) as described previously [31,32] with minor modifications. Briefly, cell culture was suspended in cell suspension buffer $(100 \mathrm{mM}$ Tris: $100 \mathrm{mM}$ EDTA, pH8.0) to $10 \mathrm{McF}$ arland standards. Proteinase $\mathrm{K}$ was then added at final concentration of $0.5 \mathrm{mg} / \mathrm{mL}$ and mixed with equal volume of molten $1 \%$ Seakem Gold Agarose before loading into the plug mold. After solidification, the agarose plugs were submerged in cell lysis buffer (CLB, $50 \mathrm{mM}$ Tris : $500 \mathrm{mM}$ EDTA, pH8.0 + 1\% sarkosyl) with lysozyme $(1 \mathrm{mg} / \mathrm{mL})$ before incubation at $37^{\circ} \mathrm{C}$ for $12 \mathrm{~h}$ and then CLB with proteinase $\mathrm{K}(0.5 \mathrm{mg} / \mathrm{mL})$ was added and cultured at $54^{\circ} \mathrm{C}$ for $2 \mathrm{~h}$ with vigorous shaking. After washing, the plugs were sliced and then digested in fresh restriction buffer with the enzyme $\operatorname{SmaI}(50 \mathrm{U} / \mu \mathrm{L})$ at $25^{\circ} \mathrm{C}$ for $12 \mathrm{~h}$. The electrophoresis was performed with CHEF-DR III system (Bio-Rad) at $14^{\circ} \mathrm{C}$ under the electric field strength $6 \mathrm{v} / \mathrm{cm}$ for $19 \mathrm{~h}$ with pulse time ramping from $2.2 \mathrm{~s}$ to $63.8 \mathrm{~s}$.

Salmonella enterica serovar Braenderup H9812 restricted with $\mathrm{XbaI}$ was used for molecular weight and size determinations. Similarities between restriction endonuclease digestion profiles were analyzed using BioNumerics software (Applied 
TABLE 1: Target genes and PCR primers used in this study.

\begin{tabular}{|c|c|c|c|}
\hline Gene target(s) & Primer sequence $\left(5^{\prime}-3^{\prime}\right)$ & Amplicon size (bp) & Reference \\
\hline \multicolumn{4}{|c|}{ Macrolide/Lincosamide resistance genes } \\
\hline \multirow{2}{*}{ ermB } & GAAAAGGTACTCAACCAAATA & \multirow{2}{*}{639} & \multirow{2}{*}{ [29] } \\
\hline & AGTAACGGTACTTAAATTGTTTAC & & \\
\hline \multirow{2}{*}{ ermA } & GAAGTTTAGCTTTCCTAA & \multirow{2}{*}{395} & \multirow{2}{*}{ [29] } \\
\hline & GCTTCAGCACCTGTCTTAATTGAT & & \\
\hline \multirow{2}{*}{$m e f A$} & AGTATCATTAATCACTAGTGC & \multirow{2}{*}{346} & \multirow{2}{*}{ [29] } \\
\hline & TTCTTCTGGTACTAAAAGTGG & & \\
\hline \multirow{2}{*}{$\ln u B$} & ССTACCTATTGTTTGTGGAA & \multirow{2}{*}{944} & \multirow{2}{*}[29]{} \\
\hline & ATAACGTTACTCTCCTATTC & & \\
\hline \multicolumn{4}{|c|}{ Tetracycline resistance genes } \\
\hline \multirow{2}{*}{ tetK } & TATTTTGGCTTTGTATTCTTTCAT & \multirow{2}{*}{1159} & \multirow{2}{*}[26]{} \\
\hline & GCTATACCTGTTCCСТCTGATAA & & \\
\hline \multirow{2}{*}{ tet $L$} & ATAAATTGTTTCGGGTCGGTAAT & \multirow{2}{*}{1077} & \multirow{2}{*}[26]{} \\
\hline & AACCAGCCAACTAATGACAATGAT & & \\
\hline \multirow{2}{*}{ tet $O$} & AACTTAGGCATTCTGGCTCAC & \multirow{2}{*}{519} & \multirow{2}{*}[26]{} \\
\hline & TCCCACTGTTCCATATCGTCA & & \\
\hline \multirow{2}{*}{ tet $M$} & GAACTCGAACAAGAGGAAAGC & \multirow{2}{*}{740} & \multirow{2}{*}[26]{} \\
\hline & ATGGAAGCCCAGAAAGGAT & & \\
\hline \multicolumn{4}{|c|}{ IntTn and xis genes } \\
\hline \multirow{2}{*}{ intTn } & GGTCTTCGTATTTCAGAGTTTGG & \multirow{2}{*}{473} & {$[30]$} \\
\hline & GTTGCATGTGCGTAATAGTTCAG & & {$[30]$} \\
\hline$x i s$ & AAGCAGACTGACATTCCTA & 193 & {$[30]$} \\
\hline & GCGTCCAATGTATCTATAA & & {$[30]$} \\
\hline & Virulence-associated factors & & \\
\hline$m r p$ & ATTGCTCCACAAGAGGATGG & 188 & {$[15]$} \\
\hline & TGAGCTTTACCTGAAGCGGT & & {$[10]$} \\
\hline$e p f$ & CGCAGACAACGAAAGATTGA & 744 & [15] \\
\hline & AAGAATGTCTTTGGCGATGG & & {$[1 \mathrm{~L}]$} \\
\hline sly & GCTTGACTTACGAGCCACAA & 248 & [15] \\
\hline & CCGCGCAATACTGATAAGC & & [15] \\
\hline$f b p$ & GACGGATCCTTTTTACATCACATGACGG & 247 & this study \\
\hline$J 0 P$ & CCGTCGACGTATTTCCGCAGAATCAT & $2+1$ & \\
\hline orf2 & CAAGTGTATGTGGATGGG & 860 & this study \\
\hline & ATCCAGTTGACACGTGCA & & \\
\hline$g d h$ & GGCGCCGAATTCGTCGACATTTAGCAATTTTTGCG & 1039 & this study \\
\hline & CGCCGCGGATCCGTAGTTAAAGTTGGTATTAAC & & \\
\hline
\end{tabular}

Maths, Kortrijk, Belgium) with Dice coefficients and clustering by an unweighted paired group with arithmetic averaging. The dendrogram of PFGE patterns of isolates tested was drawn with a $1.5 \%$ position tolerance and $1 \%$ optimization. And the cluster cutoff was set at an $85 \%$ similarity level. The different PFGE fingerprints were assigned as different uppercase letters.

2.5. Statistical Analysis. SPSS for Windows, version 16.0, was used for statistical analysis. The frequencies of antibiotic resistance, resistant genes, and virulence-associated factors were compared between the isolates from healthy carrier sows and from diseased pigs. The chi-square (or Pearson chisquare) and Fisher exact tests were used when appropriate. Differences were considered significant when two-sided $P$ value was less than 0.05 .

\section{Results}

3.1. Antimicrobial Susceptibility Testing and Detection of Resistance Genes. The collections of 96 S. suis 2 were tested for susceptibility to 12 antimicrobials (Table 2). High frequency of resistance was observed for tetracycline, followed by sulfonamides. 57 and 46 isolates from healthy sows and 28 
TABLE 2: Antimicrobial resistance profile of S. suis serotype 2 from clinically healthy carrier sows and diseased pigs.

\begin{tabular}{|c|c|c|c|c|c|c|c|c|c|}
\hline \multirow[t]{2}{*}{ Antimicrobials } & \multicolumn{3}{|c|}{$\begin{array}{l}\text { MIC breakpoint } \\
(\mu \mathrm{g} / \mathrm{mL})\end{array}$} & \multicolumn{2}{|c|}{$\begin{array}{l}\text { Healthy carrier sows } \\
\qquad(n=62)\end{array}$} & \multicolumn{2}{|c|}{$\begin{array}{l}\text { Diseased pigs } \\
\quad(n=34)\end{array}$} & \multicolumn{2}{|c|}{$\begin{array}{l}\text { Total } \\
(n=96)\end{array}$} \\
\hline & $S$ & I & $\mathrm{R}$ & MIC range & $n^{* *}$ & MIC range & $n^{* *}$ & MIC range & $n^{* *}$ \\
\hline Penicillin & 0.12 & $0.25-2$ & 4 & $\leq 0.06->8$ & 2 & $\leq 0.06-1$ & 0 & $\leq 0.06->8$ & 2 \\
\hline Ampicillin & 0.25 & $0.5-4$ & 8 & $\leq 0.12-2$ & 0 & $\leq 0.12-0.5$ & 0 & $\leq 0.12-2$ & 0 \\
\hline Erythromycin & 0.25 & 0.5 & 1 & $0.12->8$ & 35 & $\leq 0.06-4$ & 2 & $\leq 0.06->8$ & 37 \\
\hline Clindamycin & 0.5 & $1-2$ & 4 & $0.06->4$ & 35 & $0.06->4$ & 2 & $0.06->4$ & 37 \\
\hline Enrofloxacin & 0.25 & $0.5-1$ & 2 & $0.12->4$ & 6 & $0.06-1$ & 0 & $0.06->4$ & 6 \\
\hline Tetracycline & 2 & 4 & 8 & $0.5->8$ & 57 & $0.25->8$ & 28 & $0.25->8$ & 85 \\
\hline Ceftiofur & 2 & 4 & 8 & $\leq 0.12->16$ & 3 & $\leq 0.12-2$ & 0 & $\leq 0.12->16$ & 3 \\
\hline Tiamulin & 16 & - & 32 & $\leq 0.25->32$ & 7 & $\leq 0.25-16$ & 0 & $\leq 0.25->32$ & 7 \\
\hline Tilmicosin & 16 & - & 32 & $1->64$ & 35 & $\leq 0.5-64$ & 2 & $\leq 0.5->64$ & 37 \\
\hline Chloramphenicol & 4 & 8 & 16 & $\leq 1-32$ & 3 & $\leq 1-8$ & 0 & $\leq 1-32$ & 3 \\
\hline Sulfisoxazole & 256 & - & 512 & $32->512$ & 46 & $32->512$ & 18 & $32->512$ & 64 \\
\hline $\begin{array}{l}\text { Trimethoprim/ } \\
\text { sulfamethoxazole }\end{array}$ & $2 / 38$ & - & $4 / 76$ & $\begin{array}{l}\leq 0.5 / 9.5- \\
>16 / 304\end{array}$ & 13 & $\begin{array}{c}\leq 0.5 / 9.5- \\
16 / 304\end{array}$ & 2 & $\begin{array}{l}\leq 0.5 / 9.5- \\
>16 / 304\end{array}$ & 15 \\
\hline
\end{tabular}

${ }^{*}$ MIC breakpoints were taken from Clinical and Laboratory Standards Institute standards (CLSI).

S: susceptible; I: intermediate; R: resistant.

** Number of resistance isolates.

and 18 from diseased pigs were resistant to tetracycline and sulfisoxazole, respectively. These two antimicrobial agents had $\mathrm{MIC}_{50}$ values $(>8,512 \mu \mathrm{g} / \mathrm{mL}$, resp.) equal to or higher than the highest concentration tested. The lowest resistant rates of $S$. suis 2 for $\beta$-lactams were found, and all isolates were susceptible to ampicillin. Data also suggested the high incidence rates of resistance for macrolides and lincosamides in the isolates from healthy carrier sows. Among 85 tet $^{\mathrm{r}}$ isolates, 37 had $\mathrm{MLS}_{\mathrm{B}}$ resistance phenotypes, 35 from carrier sows and the remaining two from diseased pigs. No inducible resistance pattern was discovered. A Significantly higher occurrence of $\mathrm{MLS}_{\mathrm{B}}$ resistance was observed in S. suis 2 from carrier sows than those from diseased pigs $(P<0.005)$.

Antimicrobial resistance patterns and resistant determinants for tetracyclines, macrolides/lincosamides were analyzed in Table 3. No detection of tet $K$ and tet $L$ genes was observed among $\operatorname{tet}^{\mathrm{r}} \mathrm{S}$. suis 2 . The tetM gene was found among 77 and the tet $O$ gene among 51 of $85 \operatorname{tet}^{\mathrm{r}}$ S. suis type 2. None of erythromycin- and clindamycin-resistant isolates carried ermA or $\operatorname{lnuB} .35$ S. suis 2 with $\mathrm{MLS}_{\mathrm{B}}$-phenotype were shown to be ermB and tet $M$ positive and 18 mef $A$ positive. Neither ermB nor mefA was detected in the non$\mathrm{MLS}_{\mathrm{B}}$ tet $^{\mathrm{r}}$ isolates. Presence of the ermB gene was strongly associated with $\mathrm{MLS}_{\mathrm{B}}$-phenotype of $S$. suis 2. Significant carrier difference of the tet $O$ gene, but not tet $M$, was observed between erythromycin-resistant (32/37) and erythromycinsusceptible (19/48) isolates.

None of 85 tet $^{\mathrm{r}} \mathrm{S}$. suis serotype 2 was positive for the xis gene. 38 tet $^{\mathrm{r}}$ isolates (26 from diseased pigs and the remaining 12 from healthy sows) carried the intTn gene. Unexpectedly, all isolates (38/96) with intTn gene were resistant to tetracycline only, while they were susceptible to macrolides/lincosamides. And higher frequency of int Tn with tet $M$ was observed in isolates from diseased pigs (22/34) compared to those from healthy carrier sows (12/62).

3.2. Virulence-Associated Genes Analysis. 96 serotype 2 isolates were cloned and screened for the presence of the $m r p$, $e p f$, sly, orf $2, f b p$, and $g d h$ genes. The distribution of virulenceassociated genes was reported in Table 3. All isolates were positive for the virulence genes coding for $f b p$ and orf 2 and negative for $g d h .41,58$, and 47 of 62 healthy sows isolates harbored $m r p$, epf, and sly, respectively. In contrast, the $m r p$, epf, and sly genes were detected in all S. suis 2 recovered from diseased pigs but two epf-negative isolates.

Six kinds of virulence genotypes were obtained in $S$. suis capsular type 2 from carrier sows, with high frequency of $m r p+/ e p f+/ s l y+(30 / 62), m r p-/ e p f+/ s l y+(15 / 62)$, and $m r p+/ e p f+/ s l y-(9 / 62)$ (Table 4). All S. suis type 2 from diseased pigs had the virulence genotype of $m r p+/ e p f+/ s l y+$ with two exceptions of $m r p+/ e p f-/ s l y+$. Significantly lower carrier rate of $m r p+l e p f+/ s l y+$ genotype was observed among isolates from healthy sows compared to those from diseased pigs $(P<0.005)$.

3.3. PFGE Typing. On the basis of an investigation of 96 S. suis type 2, PFGE typing produced 15 different fingerprints, which were grouped into types A to $\mathrm{H}$ (Figure 1). Pulsotypes C1-C3, E1-E4, G1-G2, and H1-H2 were considered to be respectively related, with more than $85 \%$ similarity.

In contrast, significant difference was observed between the isolates from diseased pigs and healthy sows. All isolates from diseased pigs were classified as pulsotypes A, E, and $G$ (Tables 3 and 5). Pulsotypes $A$ and $E$ predominated in diseased pigs and were detected in 32 of 34 S. suis 2 isolates. For isolates from clinically healthy sows, pulsotype $G(21 / 64)$ 


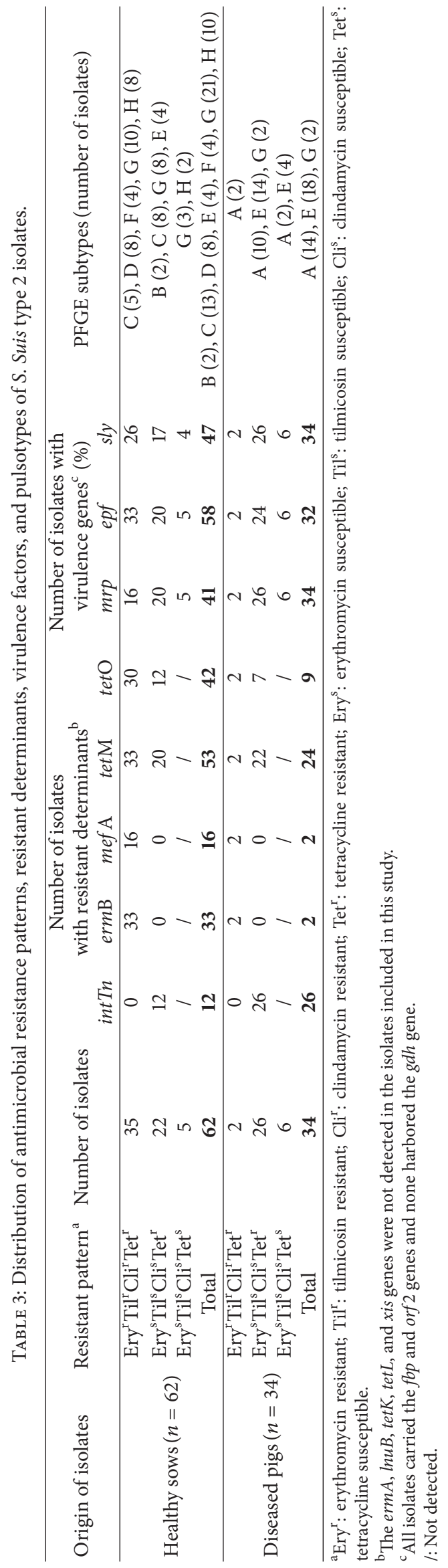


TABLE 4: Virulence genotypes of 96 S. suis capsular type 2.

\begin{tabular}{lccc}
\hline Virulence & \multicolumn{3}{c}{ Number of S. suis capsular type 2} \\
genotypes $^{*}$ & Healthy sows & Diseased pigs & Total \\
\hline mrp+/epf+/sly+ & 30 & 32 & 62 \\
$m r p+/$ epf-/sly+ & 2 & 2 & 4 \\
$m r p-/ e p f+/ s l y+$ & 15 & 0 & 15 \\
$m r p-/ e p f+/$ sly- & 4 & 0 & 4 \\
$m r p+/ e p f+/ s l y-$ & 9 & 0 & 9 \\
$m r p-/ e p f-/ s l y-$ & 2 & 0 & 2 \\
\hline Total & 62 & 34 & 96 \\
\hline
\end{tabular}

* All isolates had the genotype of fbp+/orf2+/gdh-.

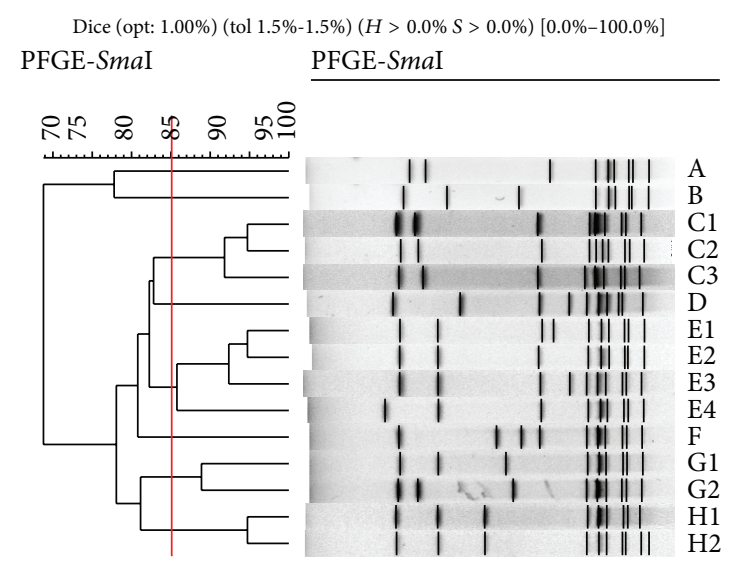

FIGURE 1: Genetic relationship of representative bands of $S$. suis type 2 isolated from clinically healthy sows and diseased pigs. Dendrogram showed the genetic relatedness of $S$. suis type 2 pulsotypes A to $\mathrm{H} 2$. The cluster cutoff (red line) was set at an $85 \%$ similarity level. Numbers at the upper left indicate percent similarity.

was the most frequently observed one, followed by types $\mathrm{C}$ (13/64), H (10/64), D (8/64), E (4/64), F (4/64), and B (2/64). Interestingly, the same pulsotype, $\mathrm{E}$ or $\mathrm{G}$, was found in $S$. suis 2 both from healthy sows and from diseased pigs. For example, isolates C55604 (from historically diseased pigs) and one isolate (farm GD-2, from healthy sows) with $100 \%$ homogeneity were present in pulsotype E2 (Table 5).

All S. suis serotype 2 from the same farm, including those isolated in different years, shared the pulsotype with more than $85 \%$ similarity; most of them had the identical PFGE patterns. 6 isolates from farm GD-1 in 2006 and 2009 were assigned to PFGE type C, and the similar results were observed for pulsotypes D, G2, H1, and E3. Complex relationship among $S$. suis 2 isolates from different farms was discovered. Three isolates from Beijing had the pulsotype (E4) different from Sichuan and Jiangsu isolates (pulsotype A) even if these S. suis serotype 2 were isolated during the largest outbreak of human $S$. suis 2 infection occurring in 2005. However, some pulsotypes were more frequently isolated and exhibited a wide distribution over herds compared to others. For instance, 13 isolates from different farms in Henan, Jiangsu, and Shandong provinces were classified as PFGE subtype G1, and similar results were
TABLE 5: PFGE patterns of 96 S. suis serotype 2 isolates included in this study.

\begin{tabular}{|c|c|c|c|c|}
\hline Origin & $\begin{array}{l}\text { PFGE pattern } \\
\left(n^{\#}\right)\end{array}$ & $\begin{array}{c}\text { Number } \\
\text { of } \\
\text { isolates }\end{array}$ & $\begin{array}{c}\text { Farm } \\
\text { number }\end{array}$ & Isolated years \\
\hline \multirow{17}{*}{$\begin{array}{l}\text { Healthy } \\
\text { sows }\end{array}$} & B (2) & 2 & $\mathrm{AH}-2$ & 2011 \\
\hline & \multirow{2}{*}{$\mathrm{C} 1(4)$} & 2 & GD-1 & 2009 \\
\hline & & 2 & GX-1 & 2007 \\
\hline & \multirow{2}{*}{ C2 (5) } & 3 & SD-1 & 2006 \\
\hline & & 2 & HB-1 & 2007 \\
\hline & C3 (4) & 4 & GD-1 & 2006, 2009 \\
\hline & \multirow{2}{*}{$\mathrm{D}(8)$} & 2 & BJ-2 & 2008 \\
\hline & & 6 & $\mathrm{HN}-3$ & 2009,2010 \\
\hline & E1 (3) & 3 & GD-2 & 2009 \\
\hline & E2 (1) & 1 & GD-2 & 2007 \\
\hline & F (4) & 4 & HB-2 & 2007 \\
\hline & \multirow{3}{*}{ G1 (13) } & 7 & $\mathrm{HN}-1$ & 2006 \\
\hline & & 1 & JS-2 & 2009 \\
\hline & & 5 & SD-2 & 2011 \\
\hline & G2 (8) & 8 & GX-2 & 2010,2012 \\
\hline & H1 (8) & 8 & JX-1 & 2007,2009 \\
\hline & $\mathrm{H} 2(2)$ & 2 & $\mathrm{SC}-3$ & 2007 \\
\hline \multirow{8}{*}{$\begin{array}{l}\text { Diseased } \\
\text { pigs }\end{array}$} & \multirow{3}{*}{ A (14) } & 8 & SC-1 & 2005 \\
\hline & & 2 & SC-2 & 2005 \\
\hline & & 4 & JS-1 & 2005 \\
\hline & $\mathrm{E} 1(2)$ & 2 & $\begin{array}{l}\text { C55609, } \\
\text { C55612 }\end{array}$ & l \\
\hline & E2 (1) & 1 & C55604 & I \\
\hline & E3 (12) & 12 & AH-1 & 2007, 2008 \\
\hline & E4 (3) & 3 & BJ-1 & 2005 \\
\hline & G1 (2) & 2 & $\mathrm{HN}-2$ & 2006 \\
\hline
\end{tabular}

\#Number of isolates with the same PFGE pattern.

\# Farm number was named as capital letters (abbreviation of the province/region)—serial number. AH: Anhui; GD: Guangdong; GX: Guangxi; SD: Shandong; HB: Hebei; BJ: Beijing; HN: Henan; SC: Sichuan; JS: Jiangsu; JX: Jiangxi. C55604, C55609, and C55612 were provided by CVCC. /: isolated time was not provided.

obtained for patterns C1, C2, and D. Furthermore, S. suis serotype 2 with types $\mathrm{G} 1, \mathrm{C} 1, \mathrm{C} 2$, and D were resistant to 
tetracycline and positive for the tet $M$ gene, although four different virulence genotypes, $m r p+/ e p f+/ s l y+, m r p-/ e p f+/ s l y+$, $m r p+/ e p f-/ s l y+$, and $m r p-/ e p f+/ s l y-$, were involved (not shown in tables).

\section{Discussion}

4.1. Resistant Phenotypes and Genotypes of S. suis 2. The lowest resistance of $S$. suis serotype 2 for $\beta$-lactams was in accordance with other discoveries $[7,8]$, supporting their use as the primary drugs to treat the infection of swine $S$. suis serotype 2 . The resistance to tetracyclines in $S$. suis has become a major worldwide problem, closely related to the widespread use of tetracycline in swine production. And tetracycline-resistance has been considered to be an important cofactor in the selection of resistance to macrolides/lincosamides [9]. In this study, 85 of the 96 isolates were resistant to tetracycline, 37 of which were coresistant to macrolides and lincosamides antibiotics. This indicated less frequent coresistance of $S$. suis 2 to tetracyclines and macrolides/lincosamides. And the similar results were also observed by other researchers $[6,10,33]$. Furthermore, 35 of 37 S. suis 2 with $\mathrm{MLS}_{\mathrm{B}}$-phenotype were isolated from healthy sows, indicating the presence of selective pressure of antimicrobial agents since tilmicosin and tylosin were widely used as swine feed additives in China. In contrast, most of tet ${ }^{\mathrm{r}}$ isolates from diseased pigs in backyard without feed additives were susceptible to macrolides/lincosamides.

Tetracyclines resistance in streptococci is mediated by ribosomal protection proteins or efflux proteins, encoded mainly by the tet genes [34]. Neither tetK nor tet $L$ was detected in this study, which was consistent with other recent analyses [10]. The tet $M$ and tet $O$ genes, both coding for ribosomal protection protein, were widespread in $\operatorname{tet}^{\mathrm{r}} S$. suis, and higher carrier rate of the tet $M$ gene than the tet $O$ gene was also observed in other studies $[9,35]$.

Resistance to macrolide of streptococcal clinical isolates is commonly encoded by ribosomal methylase ( $\mathrm{erm}$ ) genes and efflux (mef) genes [29]. The ermB gene was found in all but two erythromycin-resistant isolates, confirming its frequency in S. suis type 2 in China [9]. High number of $\mathrm{MLS}_{\mathrm{B}}$-resistant isolates with the ermB gene is in agreement with other research findings $[30,36]$. To our knowledge, no large scale survey concerning the distribution of the mefA gene in S. suis type 2 has been described in the literature. Wierzbowski et al. found that the mefA gene conferred low-level resistance of Streptococcus pneumoniae to macrolides only (M phenotype) [36]. In this study, about half of S. suis 2 with $\mathrm{MLS}_{\mathrm{B}}$ resistance harbored the mefA gene. In the view of the fact that all mefApositive isolates showed $M_{\mathrm{L}}$-phenotype and harbored the erm $B$ gene, $m e f$-mediated resistance can be obscured by the effects of the erm gene in phenotypic tests.

4.2. Tn916-Like Transposon Family. Tn916 is one of the most extensively studied conjugative transposons in grampositive bacteria. The integrase int Tn gene is responsible for transposition, and the excisase xis gene may increase the frequency of excision but is not required [37]. In this present study, it is interesting that the intTn gene was detected only in erythromycin- and clindamycin-susceptible isolates and no $x i s$ genes were detected, indicating the absence of relatedness between presence of Tn916-like conjugative transposon and macrolides/lincosamides resistance phenotypes of S. suis type 2.

It is worth noting that the tet genes are often carried by Tn916-like conjugative transposon and erythromycin resistance genes are also carried on the same element, which contributes to the coresistance of streptococci to tetracyclines and macrolides. Previous studies have investigated the association between tetM/tet $O$ and ermB/mefA in S. pneumoniae or $S$. pyogenes, and conjugal transfer experiments demonstrated that tet $M /$ tet $O$ and ermB/mefA were consistently cotransferred by Tn916-Tn1545-like transposons [38, 39]. However, the intTn gene was not detected among $35 \mathrm{MLS}_{\mathrm{B}}$ isolates with tet $M$ and ermB in this study, and therefore, elements other than the Tn916 family might be associated with coexistence of these two genes in $S$. suis serotype 2 with $M_{L} S_{B}$ phenotype.

The tetM and intTn genes, the markers for the Tn916 family of elements [40], were harbored by 22 of 36 isolates from diseased pigs in the study. Ye et al. also found that tet $M$ was associated with Tn916 in S. suis type 2 from human outbreak [9]. Thus, the presence in S. suis 2 of elements related to Tn916 with tet $M$ could play an important role in the pathogenicity of this bacterial pathogen. Further studies are necessary to monitor the spread of these elements in $S$. suis serotype 2 circulating in environments.

4.3. Virulence-Associated Factors. To further characterize the molecular features of the isolates from the diseased pigs and healthy sows, six virulence-associated genes were detected. The $g d h$ gene was not detected in any isolates, revealing that presence of this gene may be not necessary for these isolates included in this study. Different results from other studies indicated that carriage of the $g d h$ gene was associated with multilocus sequence type (ST) or origins of isolates [41]. Moreover, the $f b p$ gene was present in all S. suis type 2, and similar results were also observed by de Greeff et al. [12], who suggested that the $f b p$ gene is present among most serotypes except for serotypes 32 and 34. Thus, virulence difference between the isolates from clinically healthy sows and those from diseased pigs may lie in the frequency of the mrp, epf, and sly genes, since all $S$. suis serotype 2 analyzed in the present study also carried the orf2 gene. Interestingly, more than $60 \%$ isolates from clinically healthy sows harbored the mrp, epf, and sly genes. The higher carrier rate of virulent genotype of $m r p+/ e p f+/ s l y+$ in S. suis type 2 from diseased pigs compared to healthy sows showed that these three genes together may contribute to differentiating the virulence of serotype 2, which is in accordance with results of other epidemiological reports $[9,17]$. However, since 30 of 62 isolates from clinical carrier sows were also genotyped $m r p+/ e p f+/ s l y+$ in this study and similar result from healthy pigs was obtained by other researchers [15], it is necessary to perform further studies to specify the virulence of serotype $2 \mathrm{mrp}+/$ epf $+/ \mathrm{sly}+$ isolates. 
4.4. PFGE Subtyping. Many studies showed that PFGE could effectively detect relationship between genetic background, virulence traits, and epidemiologic implication of many bacterial pathogens [27, 31]. In this study, all isolates were typed by PFGE and clustered in 8 pulsotypes. S. suis serotype 2 from diseased pigs showed more homogeneously genetic patterns than those from healthy sows, with most of them clustering in pulsotypes $\mathrm{A}$ and $\mathrm{E}$. In contrast, the majority of isolates from healthy sows clustered in the patterns $\mathrm{G}, \mathrm{C}$, and $\mathrm{H}$, presenting a high level of divergence.

Despite the genetic diversity observed, four PFGE profiles, G, E, A, and C (accounted for 72 of 96 isolates), were more frequently observed than other patterns. These four prevalent PFGE profiles were isolated from diseased pigs and healthy sows from 9 provinces in different years (Table 5), indicating their widespread distribution in Chinese swine population. Of these, PFGE pattern G1 was unique and predominant among $S$. suis serotype 2 isolates from four epidemiologically unrelated herds, suggesting the existence of a prevalent clone. In consideration of the characteristic of their tet $M$-positive tetracycline resistance, diffusion has probably provided considerable advantages by the use of antimicrobial agents in different farms or horizontal acquiring of genetic elements, such as Tn916 with tetM $[9,42]$. In addition, S. suis serotype 2 with pulsotype G1 showed dissimilarity at virulence genotypes, including $m r p+/ e p f+/ s l y+, m r p+/ e p f-/ s l y+$, and $m r p-/ e p f+/ s l y+$, which demonstrates that factors other than antimicrobial susceptibility might have favored for its diffusion.

The identical PFGE pattern (C3, D, G2, H1, or E3) was detected for the isolates from the same farm in different years (Table 5), and the persistent dissemination of S. suis serotype 2 clone within the herd of swine could be confirmed [26]. Furthermore, pulsotype E1 (E2 or G1) was found among the isolates from historical diseased pigs and from healthy sows. In our opinion, this can be explained with two main reasons. Firstly, after a long-term adaption to the healthy sows, $S$. suis 2 gradually lost virulence and finally became avirulent. Secondly, as a kind of conditional pathogenic bacteria, the possibility that healthy carrier sows harbor S. suis 2 is capable of causing disease under specific circumstances cannot be ruled out [5], suggesting a close linkage of S. suis serotype 2 from healthy sows and diseased pigs.

Taken together, the present study is the first systematic description of resistant phenotypes and genetic genotypes of $S$. suis type 2 isolated from clinically healthy sows and diseased pigs in China. Significant differences of $M_{B}$ resistance phenotype, virulence-associated genotypes, and PFGE pulsotypes were observed between isolates from clinical carrier sows and those from diseased pigs. The results indicate that $\beta$-lactams are still the primary drugs to treat the infection of swine $S$. suis serotype 2 . The unique and predominant PFGE types within and between herds show persistent dissemination of S. suis 2 and a close linkage among isolates from healthy sows and diseased pigs. Moreover, our data also support the contention that extensive use of tetracycline and horizontal acquiring of genetic element, Tn916 with tetM, could act as a selective factor for the pathogenicity and widespread diffusion of serotype $2[9,42]$.

\section{Conflict of Interests}

The authors declare that there is no conflict of interests regarding the publication of this paper.

\section{Acknowledgments}

This study was supported by NSF of China (31302142), Beijing NOVA Program (2009 B 52), and 948 Program (2011-G14) from MOA of China.

\section{References}

[1] M. Gottschalk, M. Segura, and J. Xu, "Streptococcus suis infections in humans: the Chinese experience and the situation in North America," Animal Health Research Reviews, vol. 8, no. 1, pp. 29-45, 2007.

[2] M. Fulde and P. Valentin-Weigand, "Epidemiology and pathogenicity of zoonotic streptococci," Current Topics in Microbiology and Immunology, vol. 368, pp. 49-81, 2013.

[3] K. Maneerat, S. Yongkiettrakul, I. Kramomtong et al., "Virulence genes and genetic diversity of Streptococcus suis serotype 2 isolates from Thailand," Transboundary and Emerging Diseases, vol. 60, no. 2, pp. 69-79, 2013.

[4] T. C. Smith, A. W. Capuano, B. Boese, K. P. Myers, and G. C. Gray, "Occupational exposure to Streptococcus suis among US swine workers," Emerging Infectious Diseases, vol. 14, no. 12, pp. 1925-1927, 2008.

[5] J. J. Staats, I. Feder, O. Okwumabua, and M. M. Chengappa, "Streptococcus suis: past and present," Veterinary Research Communications, vol. 21, no. 6, pp. 381-407, 1997.

[6] A. I. Vela, M. A. Moreno, J. A. Cebolla et al., "Antimicrobial susceptibility of clinical strains of Streptococcus suis isolated from pigs in Spain," Veterinary Microbiology, vol. 105, no. 2, pp. 143-147, 2005.

[7] H. J. Wisselink, K. T. Veldman, C. van den Eede, S. A. Salmon, and D. J. Mevius, "Quantitative susceptibility of Streptococcus suis strains isolated from diseased pigs in seven European countries to antimicrobial agents licenced in veterinary medicine," Veterinary Microbiology, vol. 113, no. 1-2, pp. 73-82, 2006.

[8] C. Zhang, Y. Ning, Z. Zhang, L. Song, H. Qiu, and H. Gao, "In vitro antimicrobial susceptibility of Streptococcus suis strains isolated from clinically healthy sows in China," Veterinary Microbiology, vol. 131, no. 3-4, pp. 386-392, 2008.

[9] C. Ye, X. Bai, J. Zhang et al., "Spread of Streptococcus suis sequence type 7, China," Emerging Infectious Diseases, vol. 14, no. 5, pp. 787-791, 2008.

[10] Y. W. Chu, T. K. M. Cheung, M. Y. Chu et al., "Resistance to tetracycline, erythromycin and clindamycin in Streptococcus suis serotype 2 in Hong Kong," International Journal of Antimicrobial Agents, vol. 34, no. 2, pp. 181-182, 2009.

[11] J. Brassard, M. Gottschalk, and S. Quessy, "Cloning and purification of the Streptococcus suis serotype 2 glyceraldehyde-3phosphate dehydrogenase and its involvement as an adhesin," Veterinary Microbiology, vol. 102, no. 1-2, pp. 87-94, 2004.

[12] A. de Greeff, H. Buys, R. Verhaar, J. Dijkstra, L. van Alphen, and H. E. Smith, "Contribution of fibronectin-binding protein to pathogenesis of Streptococcus suis serotype 2," Infection and Immunity, vol. 70, no. 3, pp. 1319-1325, 2002.

[13] S. J. King, P. J. Heath, I. Luque, C. Tarradas, C. G. Dowson, and A. M. Whatmore, "Distribution and genetic diversity of suilysin 
in Streptococcus suis isolated from different diseases of pigs and characterization of the genetic basis of suilysin absence," Infection and Immunity, vol. 69, no. 12, pp. 7572-7582, 2001.

[14] R. Kutz and O. Okwumabua, "Differentiation of highly virulent strains of Streptococcus suis serotype 2 according to glutamate dehydrogenase electrophoretic and sequence type," Journal of Clinical Microbiology, vol. 46, no. 10, pp. 3201-3207, 2008.

[15] L. M. G. Silva, C. G. Baums, T. Rehm, H. J. Wisselink, R. Goethe, and P. Valentin-Weigand, "Virulence-associated gene profiling of Streptococcus suis isolates by PCR," Veterinary Microbiology, vol. 115, no. 1-3, pp. 117-127, 2006.

[16] H. E. Smith, U. Vecht, H. J. Wisselink, N. Stockhofe-Zurwieden, Y. Biermann, and M. A. Smits, "Mutants of Streptococcus suis types 1 and 2 impaired in expression of muramidase-released protein and extracellular protein induce disease in newborn germfree pigs," Infection and Immunity, vol. 64, no. 10, pp. 44094412, 1996.

[17] Z. Wei, R. Li, A. Zhang et al., "Characterization of Streptococcus suis isolates from the diseased pigs in China between 2003 and 2007," Veterinary Microbiology, vol. 137, no. 1-2, pp. 196-201, 2009.

[18] L. Bonifait, K. Vaillancourt, M. Gottschalk, M. Frenette, and D. Grenier, "Purification and characterization of the subtilisin-like protease of Streptococcus suis that contributes to its virulence," Veterinary Microbiology, vol. 148, no. 2-4, pp. 333-340, 2011.

[19] Y. Pian, S. Gan, S. Wang et al., "Fhb, a novel factor H-binding surface protein, contributes to the antiphagocytic ability and virulence of streptococcus suis," Infection and Immunity, vol. 80, no. 7, pp. 2402-2413, 2012.

[20] G. Martinez, J. Harel, S. Lacouture, and M. Gottschalk, "Genetic diversity of Streptococcus suis serotypes 2 and 1/2 isolates recovered from carrier pigs in closed herds," Canadian Journal of Veterinary Research, vol. 66, no. 4, pp. 240-248, 2002.

[21] S. R. Rasmussen, F. M. Aarestrup, N. E. Jensen, and S. E. Jorsal, "Associations of Streptococcus suis serotype 2 ribotype profiles with clinical disease and antimicrobial resistance," Journal of Clinical Microbiology, vol. 37, no. 2, pp. 404-408, 1999.

[22] S. J. King, J. A. Leigh, P. J. Heath et al., "Development of a multilocus sequence typing scheme for the pig pathogen Streptococcus suis: identification of virulent clones and potential capsular serotype exchange," Journal of Clinical Microbiology, vol. 40, no. 10, pp. 3671-3680, 2002.

[23] C. Ye, X. Zhu, H. Jing et al., "Streptococcus suis sequence type 7 outbreak, Sichuan, China," Emerging Infectious Diseases, vol. 12, no. 8, pp. 1203-1208, 2006.

[24] Z. Wu, M. Li, C. Wang et al., "Probing genomic diversity and evolution of Streptococcus suis serotype 2 by NimbleGen tiling arrays," BMC Genomics, vol. 12, article 219, 2011.

[25] F. Berthelot-Hérault, C. Marois, M. Gottschalk, and M. Kobisch, "Genetic diversity of Streptococcus suis strains isolated from pigs and humans as revealed by pulsed-field gel electrophoresis," Journal of Clinical Microbiology, vol. 40, no. 2, pp. 615-619, 2002.

[26] M. S. Princivalli, C. Palmieri, G. Magi et al., "Genetic diversity of Streptococcus suis clinical isolates from pigs and humans in Italy (2003-2007)," Euro Surveillance, vol. 14, no. 33, 2009.

[27] A. I. Vela, J. Goyache, C. Tarradas et al., "Analysis of genetic diversity of Streptococcus suis clinical isolates from pigs in Spain by pulsed-field gel electrophoresis," Journal of Clinical Microbiology, vol. 41, no. 6, pp. 2498-2502, 2003.

[28] Clinical and Laboratory Standards Institute, Performance Standards for Antimicrobial Disk and Dilution Susceptibility Tests for Bacteria Isolated from Animals, vol. 28, Wayne, Pa, USA, 3rd edition, 2008.
[29] S. E. Gygax, J. A. Schuyler, L. E. Kimmel, J. P. Trama, E. Mordechai, and M. E. Adelson, "Erythromycin and clindamycin resistance in group B streptococcal clinical isolates," Antimicrobial Agents and Chemotherapy, vol. 50, no. 5, pp. 1875-1877, 2006.

[30] E. Pérez-Trallero, M. Montes, B. Orden, E. Tamayo, J. M. GarcíaArenzana, and J. M. Marimón, "Phenotypic and genotypic characterization of Streptococcus pyogenes isolates displaying the MLSB phenotype of macrolide resistance in Spain, 1999 to 2005," Antimicrobial Agents and Chemotherapy, vol. 51, no. 4, pp. 1228-1233, 2007.

[31] R. K. Gautom, "Rapid pulsed-field gel electrophoresis protocol for typing of Escherichia coli O157:H7 and other gram-negative organisms in 1 day," Journal of Clinical Microbiology, vol. 35, no. 11, pp. 2977-2980, 1997.

[32] C. K. Luey, Y. W. Chu, T. K. Cheung et al., "Rapid pulsed-field gel electrophoresis protocol for subtyping of Streptococcus suis serotype 2," Journal of Microbiological Methods, vol. 68, no. 3, pp. 648-650, 2007.

[33] J. Marie, H. Morvan, F. Berthelot-Hérault et al., "Antimicrobial susceptibility of Streptococcus suis isolated from swine in France and from humans in different countries between 1996 and 2000," Journal of Antimicrobial Chemotherapy, vol. 50, no. 2, pp. 201-209, 2002.

[34] B. S. Speer, N. B. Shoemaker, and A. A. Salyers, "Bacterial resistance to tetracycline: mechanisms, transfer, and clinical significance," Clinical Microbiology Reviews, vol. 5, no. 4, pp. 387-399, 1992.

[35] Y. Tian, F. M. Aarestrup, and C. P. Lu, "Characterization of Streptococcus suis serotype 7 isolates from diseased pigs in Denmark," Veterinary Microbiology, vol. 103, no. 1-2, pp. 55-62, 2004.

[36] A. K. Wierzbowski, K. Nichol, N. Laing et al., "Macrolide resistance mechanisms among streptococcus pneumoniae isolated over 6 years of Canadian Respiratory Organism Susceptibility Study (CROSS) (1998-2004)," Journal of Antimicrobial Chemotherapy, vol. 60, no. 4, pp. 733-740, 2007.

[37] L. B. Rice, "Tn916 family conjugative transposons and dissemination of antimicrobial resistance determinants," Antimicrobial Agents and Chemotherapy, vol. 42, no. 8, pp. 1871-1877, 1998.

[38] M. P. Montanari, I. Cochetti, M. Mingoia, and P. E. Varaldo, "Phenotypic and molecular characterization of tetracyclineand erythromycin-resistant strains of Streptococcus pneumoniae," Antimicrobial Agents and Chemotherapy, vol. 47, no. 7, pp. 2236-2241, 2003.

[39] A. Brenciani, A. Bacciaglia, M. Vecchi, L. A. Vitali, P. E. Varaldo, and E. Giovanetti, "Genetic elements carrying erm(B) in Streptococcus pyogenes and association with tet $(\mathrm{M})$ tetracycline resistance gene," Antimicrobial Agents and Chemotherapy, vol. 51, no. 4, pp. 1209-1216, 2007.

[40] P. Spigaglia, F. Barbanti, and P. Mastrantonio, "New variants of the tet $(\mathrm{M})$ gene in Clostridium difficile clinical isolates harbouring Tn916-like elements," Journal of Antimicrobial Chemotherapy, vol. 57, no. 6, pp. 1205-1209, 2006.

[41] A. Kerdsin, S. Dejsirilert, P. Puangpatra et al., "Genotypic profile of Streptococcus suis serotype 2 and clinical features of infection in humans, Thailand," Emerging Infectious Diseases, vol. 17, no. 5, pp. 835-842, 2011.

[42] I. Klare, C. Konstabel, D. Badstübner, G. Werner, and W. Witte, "Occurrence and spread of antibiotic resistances in Enterococcus faecium," International Journal of Food Microbiology, vol. 88, no. 2-3, pp. 269-290, 2003. 

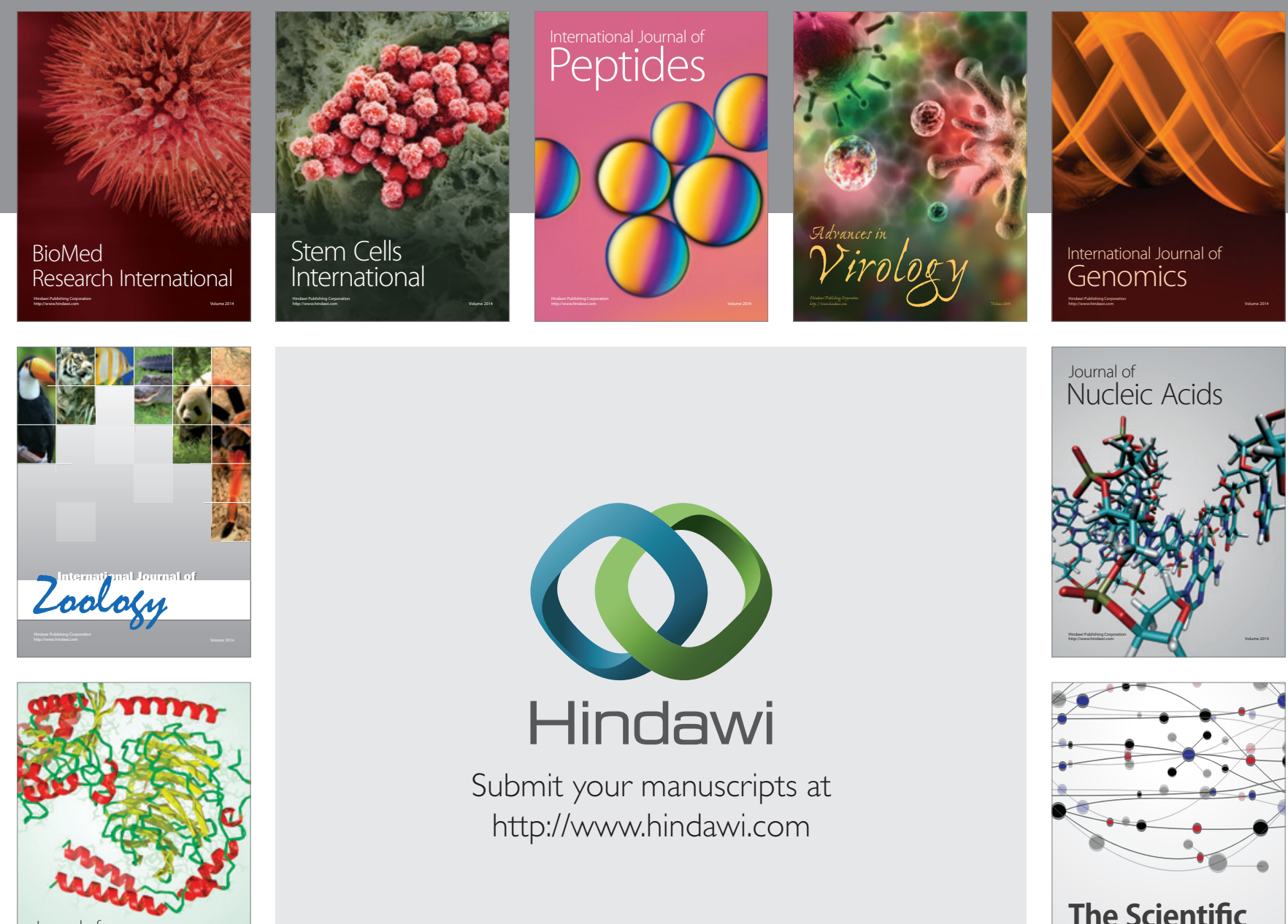

Submit your manuscripts at

http://www.hindawi.com

Journal of
Signal Transduction
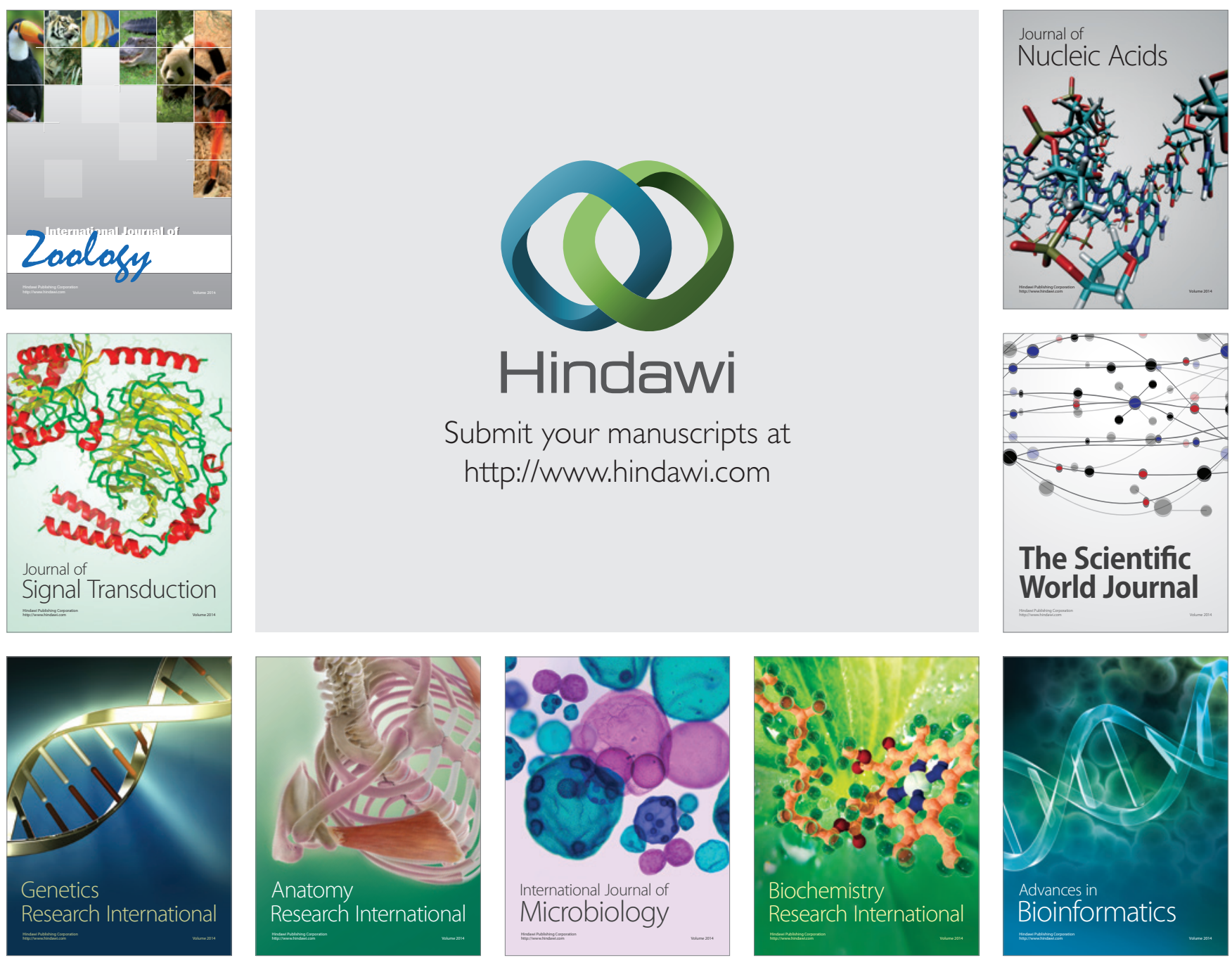

The Scientific World Journal
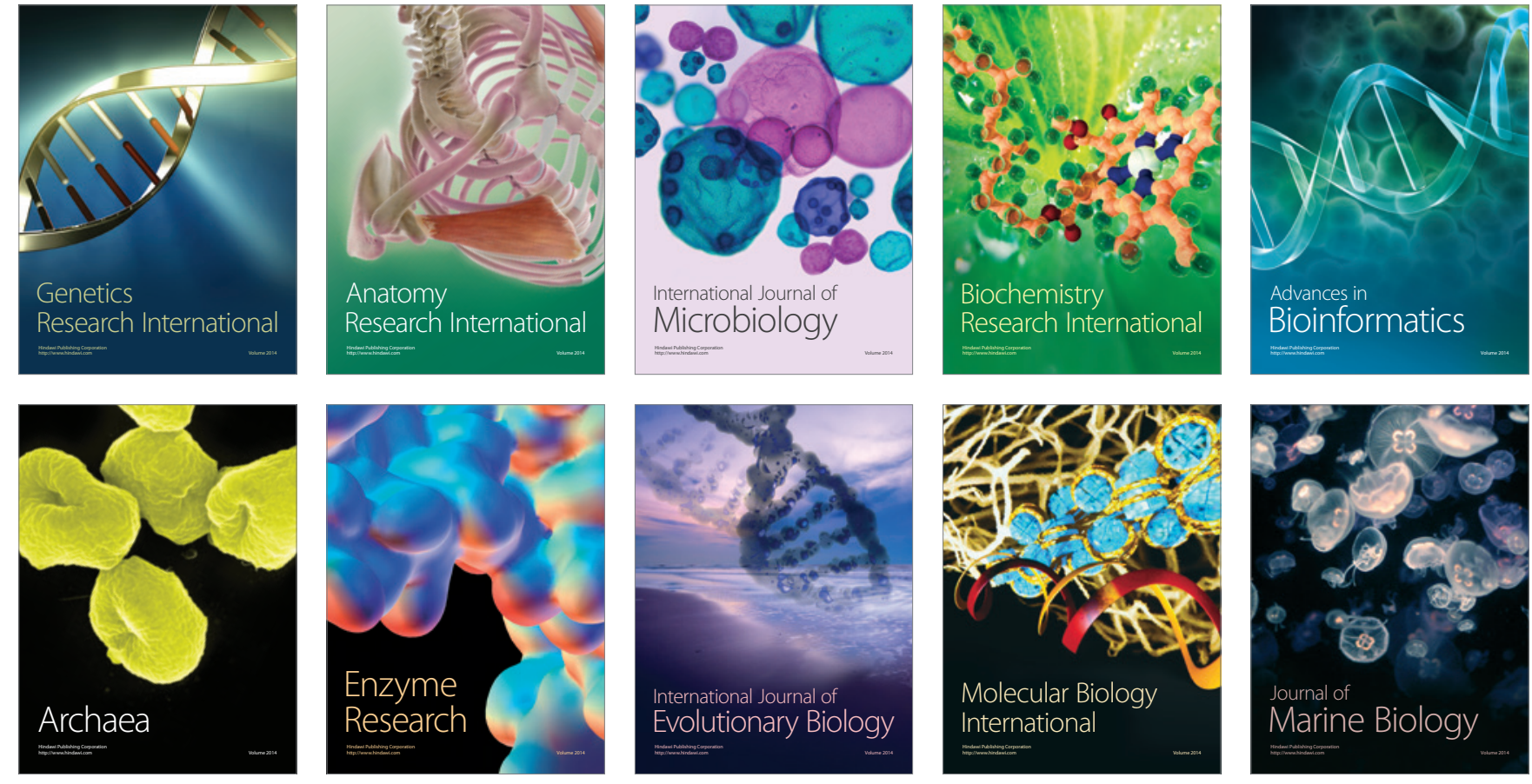